\title{
Pachychoroid Neovasculopathy Disguising as Age-Related Macular Degeneration Treated by Spironolactone and Anti-VEGF Combination Therapy
}

\author{
Leonie F. Keidel Benedikt Schworm Siegfried G. Priglinger \\ Jakob Siedlecki
}

Department of Ophthalmology, Ludwig-Maximilians-University, Munich, Germany

\author{
Keywords \\ Central serous chorioretinopathy · Age-related macular degeneration - Spironolactone . \\ Choroid · Tomography · Optical coherence
}

\begin{abstract}
Nonresponse of neovascular age-related macular degeneration (nAMD) to anti-vascular endothelial growth factor (anti-VEGF) therapy can often be attributed to misdiagnosis, and pathologies mimicking AMD might require different therapeutic concepts. In the following, we want to outline a case of presumed nAMD which revealed to be pachychoroid neovasculopathy (PNV) and was successfully treated by the addition of spironolactone. A 67-year-old female patient was referred for nonresponse of nAMD on her left eye after 29 intravitreal injections of aflibercept with no complete resolution of subretinal fluid. On fundoscopy, both maculae presented with pigment epithelium alterations, while the left eye showed subretinal fluid on optical coherence tomography (OCT) with an associated pigment epithelium detachment, which revealed to contain a neovascular network on OCT angiography. There was faint leakage on fluorescence (FAG) and indocyanine green angiography (ICGA) and some focal vascular dilation of the neovascular network on ICGA. Due to the absence of Drusen on any eye, a thick choroid, and the presence of a gravitational tract on blue autofluorescence (BAF), chronic central serous chorioretinopathy with a choroidal neovascularization, defined as PNV in the pachychoroid disease was diagnosed. Upon the addition of spironolactone to anti-VEGF treatment, choroidal thickness significantly decreased, and subretinal fluid resolution was observed and maintained for the first time. In conclusion, PNV should be ruled out in cases of presumed nAMD nonresponding to anti-VEGF. In these cases, a combination therapy of anti-VEGF and mineralocorticoid antagonists can facilitate fluid resorption.
\end{abstract}

\section{Karger $\stackrel{2}{\circ}$}




\section{Introduction}

Nonresponse of neovascular age-related macular degeneration (nAMD) to intravitreal vascular endothelial growth factor (VEGF) inhibition affects approximately 5-10\% of eyes and is attributable to misdiagnosis in almost $50 \%$ of cases [1]. Therefore, cases of primarily supposed neovascular AMD should be reevaluated for correct diagnosis, if anti-VEGF therapy proves to be nonefficient $[1,2]$.

The recently established pachychoroid spectrum of macular disorders has been shown to represent an important differential diagnosis to nAMD [3]. Pachychoroid is defined as subfoveal diffuse or focal increase in choroidal thickness $(>300 \mu \mathrm{m})$ on optical coherence tomography (OCT), which is typically associated with abnormally dilated Haller layer vessels (pachyvessels) and an attenuation of the inner choroid (Sattler's layer and choriocapillaris) [3]. Long-term anti-VEGF treatment may induce choroidal thinning of the former above norm thickened choroid [4]. Symptomatic pachychoroid means the presence of either pachychoroidal pigment epitheliopathies, central serous chorioretinopathy (CSC), pachychoroid neovasculopathy (PNV) or pachychoroid aneurysmal type 1 choroidal neovascularization (CNV) (formerly polypoidal choroidal vasculopathy) (PAT1). CSC is present, when a serous retinal detachment with or without serous pigment epithelial detachment (PED), could be visualized. Typical changes like an RPE leakage and delayed choroidal filling in fluorescein and indocyanine green angiography (ICGA) must have been detectable. In $25 \%$ of eyes with CSC a PNV, a CNV formation, atop of a thickened choroid develops. It can be visualized on OCT and confirmed by fluorescein and ICGA with evidence of RPE atrophy, diffuse late leakage, and a late staining plaque on ICGA. Changes are considered a PAT1 when RPE detachments associated with choroidal polypoidals were present on OCT and ICGA showed the typical branching vascular network appearance with terminal aneurysmal dilatations.

Although nAMD and the pachychoroid disease spectrum feature completely contrary etiopathologies - a pachychoroid due to faulty choroidal autoregulation versus degenerative retinal pigment epithelium and choroidal atrophy in AMD, the distinction between both entities can sometimes be difficult [3]. This is especially true in older patients showing chronic courses of pachychoroid disease and in cases complicated by CNV and PAT1 [5, 6].

In the following, we would like to outline a case of presumed neovascular AMD with nonresolving subretinal fluid refractory to anti-VEGF, which revealed to be a pachychoroid spectrum disorder complicated by CNV on multimodal imaging. In addition to continued antiVEGF therapy, we report on the therapeutic addition of the mineralocorticoid antagonist spironolactone, which was used to reduce choroidal thickness and successfully eliminate subretinal fluid previously unresponsive to anti-VEGF.

\section{Case Report}

A 67-year-old patient with a history of chronic foveal PED and subretinal fluid on the left eye interpreted as neovascular AMD ex domo was referred to our clinic due to nonresponse to anti-VEGF therapy. On her left eye, 29 monthly aflibercept (Bayer AG, Leverkusen, Germany) injections had been performed for 29 months prior to presentation without achieving complete resolution of subretinal fluid. During the course of the illness, there had never been any signs of intraretinal fluid on OCT. She stated that the onset of first visual symptoms had been at the age of 40 without any medical treatment until commencement of anti-VEGF. Her right eye had a history of foveal pigment alteration, which over the course of the past years had developed a shallow PED and transient subretinal fluid, which showed complete reso-

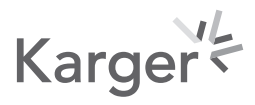


lution of subretinal fluid after 1 intravitreal injection of ranibizumab (Lucentis, Novartis Pharma, Basel, Switzerland) 22 months before presentation.

Her best-corrected visual acuity was 20/80 on the left eye and 20/32 on the right eye, respectively. Except from a beginning corticonuclear cataract, there were no pathological findings on anterior segment examination on both eyes. Intraocular pressure was normal. There was no family history of any kind of retinal pathology. Medical history included arterial hypertension, hypothyroidism, arthritis, and psoriasis. Medication consisted of ramipril 2.5 $\mathrm{mg}$, bisoprolol $2.5 \mathrm{mg}$, torasemide $10 \mathrm{mg}$, and L-Thyroxine $0.1 \mathrm{mg}$.

On fundoscopy, both maculae presented with pigment epithelium alterations, while the left macula was slightly prominent. In the following, multimodal imaging including (enhanced depth, EDI) spectral-domain OCT (SD-OCT), near-infrared (NIR) imaging, blue autofluorescence (BAF) confocal laser scanning ophthalmology, fluorescein (FAG), and indocyanine green angiography (ICGA) were performed, all using Spectralis HRA + OCT (Heidelberg Engineering, Heidelberg, Germany). Additionally, OCT angiography (OCT-A) was performed using Angioplex on the Cirrus 5000 OCT (Carl Zeiss Meditec AG, Jena, Germany).

On NIR and SD-OCT, the right eye presented with pigment epithelium mottling and a shallow PED, which showed a type 1 CNV on OCT-A without leakage on FAG/ICGA and OCT-A. Multifocal choroidal hyperpermeability was seen on ICGA. On EDI SD-OCT, choroidal thickness was $371 \mu \mathrm{m}$ subfoveally.

On NIR and SD-OCT, the left eye presented with a serous neurosensory detachment of the fovea with elongated photoreceptors and an underlying shallow, irregular, vascularized PED (shown in Fig. 1a). Mean subfoveal choroidal thickness was $266 \mu \mathrm{m}$ (shown in Fig. 1a). An illdefined gravitational tract of 4,322 $\mu \mathrm{m}$ diameter horizontally and 4,844 $\mu \mathrm{m}$ vertically was seen on BAF (shown in Fig. 1b). The presence of a CNV type 1 was confirmed on FAG/ICGA and OCT-A (shown in Fig. 2). Only faint leakage was observed from the CNV.

Due to the absence of Drusen on any eye, the findings of multimodal imaging and the nonresponse to anti-VEGF, chronic CSC complicated by a secondary CNV - or, as suggested by the new pachychoroid terminology, PNV - was suspected. As some focal vascular dilations of the neovascular network were visible on ICGA of the left eye, inactive polyps after long-term anti-VEGF therapy as part of a PAT1 cannot be excluded. We did not see nodular flow in either en face or cross-sectional OCT-A scans to confirm active PAT1 [7].

All possible further options, including photodynamic therapy, were discussed with the patient, who preferred a temporary cessation of anti-VEGF therapy and the start of off-label
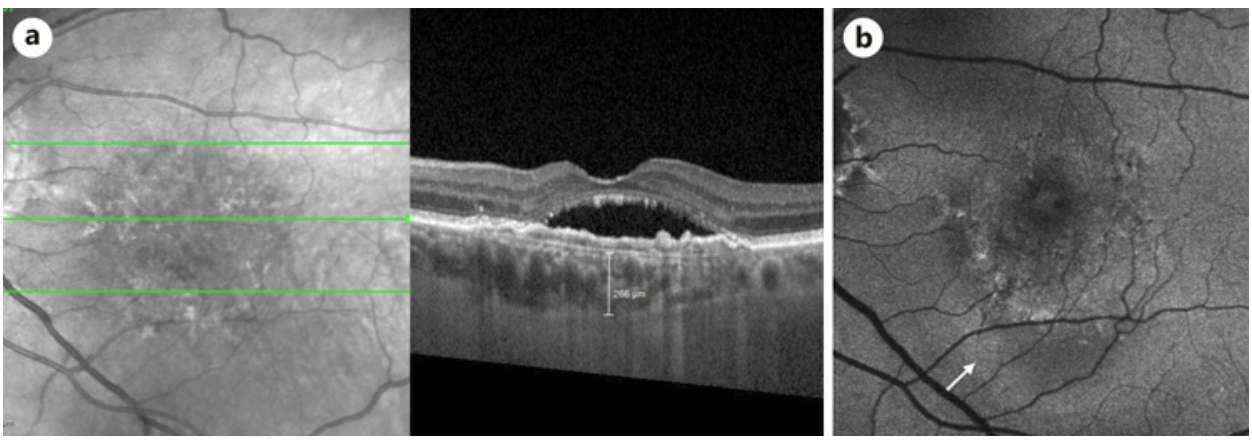

Fig. 1. Multimodal imaging specifically for diseases of the pachychoroid disease spectrum. On EDI-OCT (a) thinning in sattler's and choriocapillaris layer, pathologically dilated veins in the Haller's layer and increased choroidal thickening can be visualized. b BAF reveals a shallow hyper-reflectivity inferior to the fovea, indicating the presence of former subretinal fluid accumulation forming a gravitational tract ( $\operatorname{arrowhead} \Delta$ ). OCT, optical coherence tomography; BAF, blue autofluorescence. 
Keidel et al.: Combination Therapy for Pachychoroid Neovasculopathy Disguising as AMD

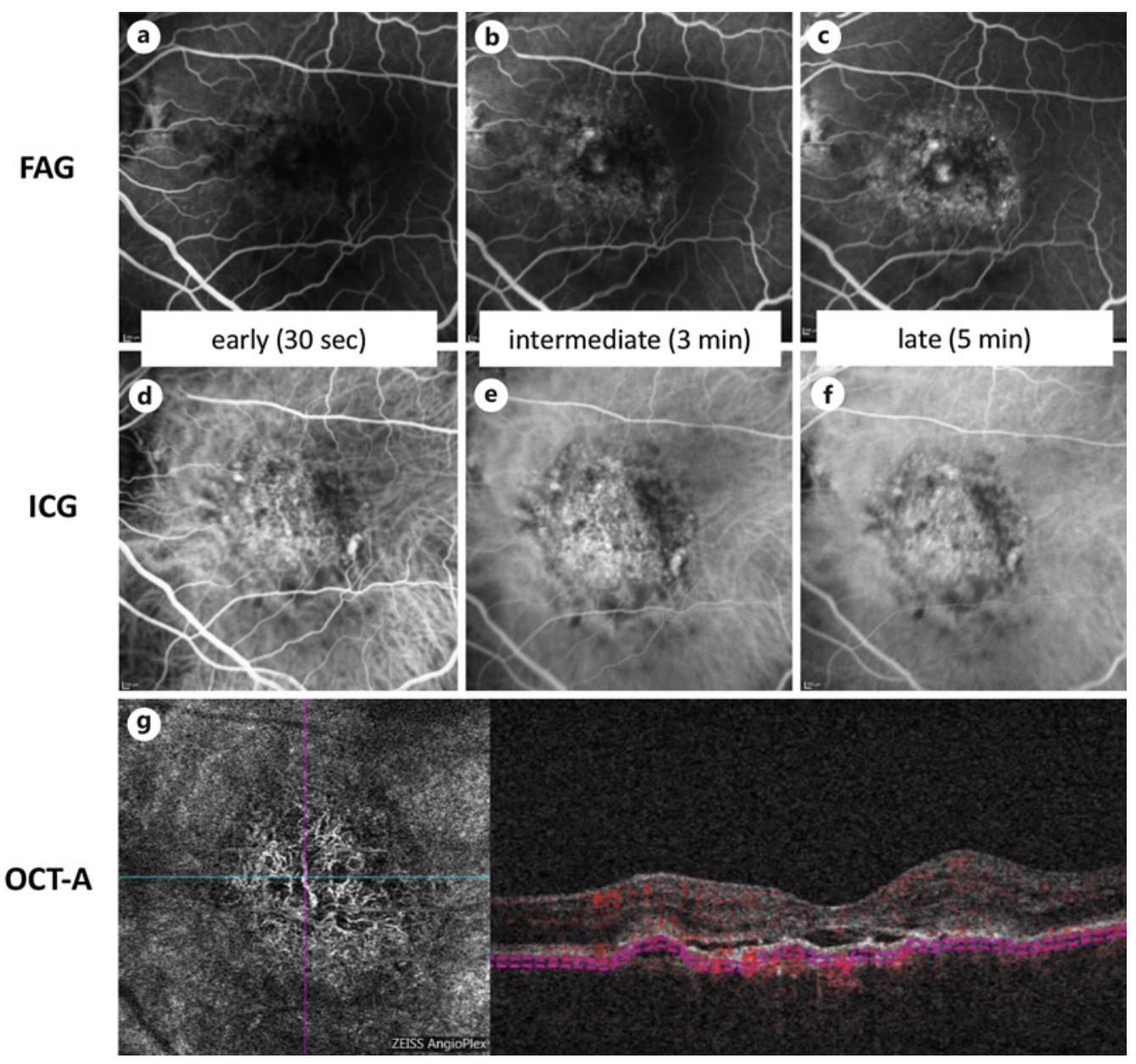

Fig. 2. Vessel imaging. On fluorescein angiography (a-c), only faint late leakage and mostly staining can be visualized. On ICG (d-f) and even better on OCT-A (g), the borders of the type 1 CNV can be visualized. OCT, optical coherence tomography; OCT-A, OCT angiography; ICG, indocyanine green angiography.

spironolactone $25 \mathrm{mg}$ BID. Within 6 weeks, subfoveal fluid significantly diminished by 51\% from 108 to $55 \mu \mathrm{m}$ (shown in Fig. 3b) and by $90 \%$ to $12 \mu \mathrm{m}$ after 12 weeks (shown in Fig. 3c). The same applied for choroidal thickness, which decreased from $366 \mu \mathrm{m}$ by $19 \%$ to $295 \mu \mathrm{m}$ at 6 weeks and by $27 \%$ to $267 \mu \mathrm{m}$ at 12 weeks. Simultaneously to the reduction in subretinal fluid and choroidal thickness, at week 6 the PED was noted to grow on the nasal and temporal CNV borders in the parafoveal region, partially penetrating the retinal pigment epithelium, defining conversion into a type $2 \mathrm{CNV}$ (shown in Fig. 3b). Until the next follow-up after 12 weeks (shown in Fig. 3c), CNV growth and RPE penetration continued, along with newly diagnosed subretinal hyper-reflective material atop the type II CNV borders (shown in Fig. 3c). FAG and OCT-A confirmed the diagnosis of a type II CNV.

Twelve weeks after stopping anti-VEGF and commencing spironolactone, spironolactone was stopped and aflibercept administered again. As a consequence, the type 2 segments of the CNV regressed below the RPE, the PED returned to its original configuration, and subretinal fluid disappeared (shown in Fig. 3d). After 3 further injections, subfoveal choroidal thickness had additionally decreased by $20 \%$ to $214 \mu \mathrm{m}$, resulting in a total reduction by $42 \%$ from 366 $\mu \mathrm{m}$ at baseline. Due to long-standing disease, the inner foveal thickness remained thinned at $75 \mu \mathrm{m}$. Final visual acuity was $20 / 50$. 


\section{Case Reports in Ophthalmology}

\begin{tabular}{l|l}
\hline Case Rep Ophthalmol 2021;12:116-123 \\
\hline DOI: 10.1159/000510828 & $\begin{array}{l}\text { (c) 2021 The Author(s). Published by S. Karger AG, Basel } \\
\text { www.karger.com/cop }\end{array}$ \\
\hline
\end{tabular}

Keidel et al.: Combination Therapy for Pachychoroid Neovasculopathy Disguising as AMD
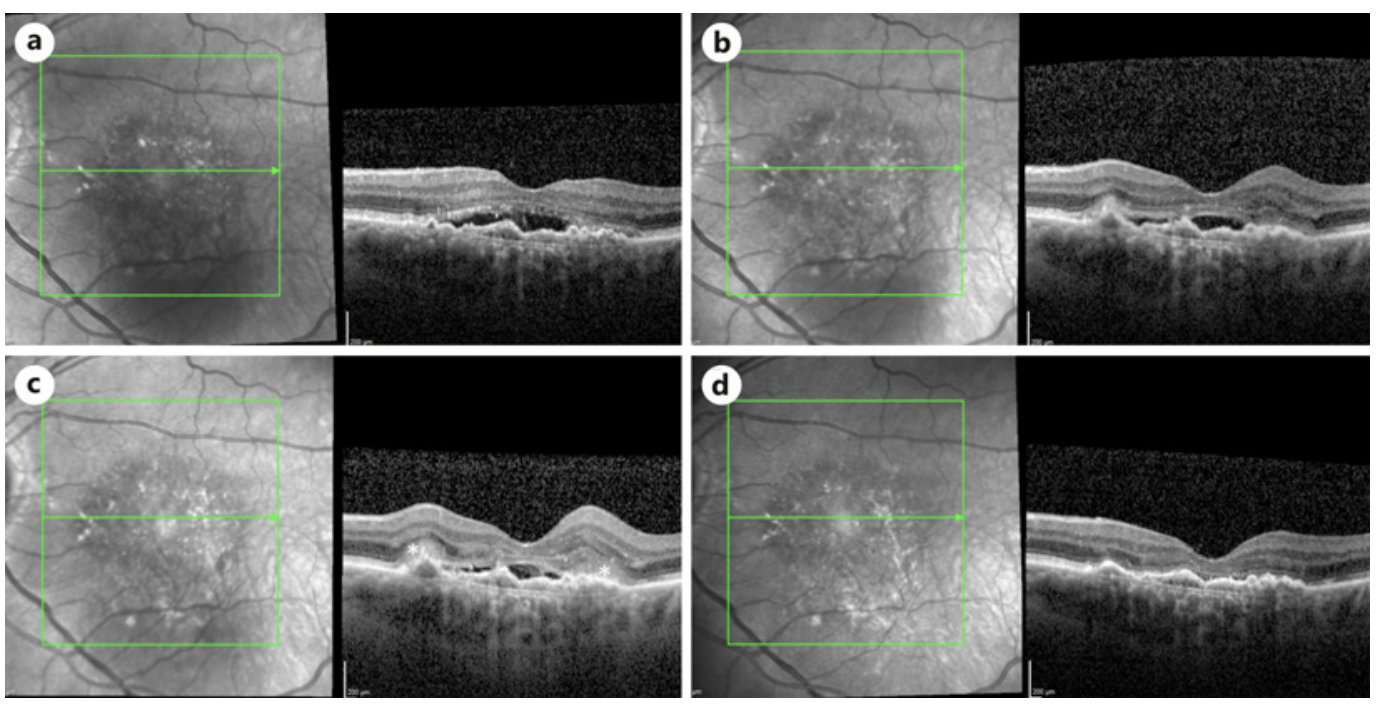

Fig. 3. Time course of central retinal thickness, subretinal fluid accumulation, and structural PED changes on OCT. a OCT on the day of presentation (after 29 aflibercept injections) shows a serous detachment of the fovea with elongated photoreceptors and an underlying shallow PED. b OCT 6 weeks after spironolactone administration shows a decrease in subfoveal fluid by 51\%. PED growth was noted on the nasal and temporal CNV borders in the parafoveal region. c OCT 12 weeks after spironolactone administration shows a further decrease in subfoveal fluid by $90 \%$, while further CNV growth at the PED borders can be noted, partially penetrating the retinal pigment epithelium with deposition of SHRM $\left(^{*}\right)$ atop the now type II CNV borders. d OCT 17 weeks after spironolactone administration and 5 weeks after a further anti-VEGF (aflibercept) injection, a complete resolution of subretinal fluid is achieved. anti-VEGF, anti-vascular endothelial growth factor; PED, pigment epithelium detachment; OCT, optical coherence tomography; SHRM, subretinal hyper-reflective material.

\section{Discussion}

Since the introduction of OCT, the diagnosis of CSC has been mainly based on the presence of foveal subretinal fluid causing neurosensory detachment [3]. Nowadays, enhanced depth OCT imaging, however, allows for additional visualization of the choroid, and a multitude of diseases, including CSC, have been shown to feature overlapping, characteristic alterations of the choroidal vascular architecture [3]. As introduced by Warrow and colleagues [8], the term pachychoroid is increasingly being used to summarize this set of newly characterized macular disorders, which all feature choroidal thickening and hyperperfusion, but can be defined as single entities - or 1 disease in different stages - by the type and amount of other macular tissues affected by the choroidal malfunction (e.g., retinal pigment epithelium, outer retina, and CNV).

The pachychoroid spectrum is increasingly recognized as a frequent cause of misdiagnosis in AMD. Theoretically, the presence of Drusen is a certain pathognomonic finding of AMD, which in return should exclude the diagnosis of AMD in their absence. However, Drusen can regress upon the formation of CNV or macular atrophy caused by either disorder; type 2 $\mathrm{CNV}$ can lead to such severe fibrovascular disruption of the retina that Drusen as underlying cause of the $\mathrm{CNV}$ can be obscured; and type $1 \mathrm{CNV}$ caused by pachychoroid diseases can mimic Drusen [9]. In 2012, Fung and colleagues [2] already reported about the phenotypical similarity of type $1 \mathrm{CNV}$ in chronic CSC and neovascular AMD. In more recent reports on macular morphology in the CATT trials, Jaffe and colleagues [8] noted that the presence of subfoveal fluid was a consistent biomarker of good visual acuity in neovascular AMD throughout years 1-5 under anti-VEGF therapy, and recently hypothesized that this might be partly caused by 
associated pachychoroid disease providing better long-term prognosis than classic neovascular AMD. In contrast to nAMD, in the pachychoroid disease spectrum, CNV is thought to be caused by an increased choroidal perfusion leading to stress in the endothelial cells of the choriocapillaris and resulting in extracellular remodeling (arteriogenesis). As a next step, arteriogenesis-derived vessels may evolve above the destructed RPE cells and form a type I $\mathrm{CNV}$. As recently suggested by Lupidi et al. [10] the loss of the adaptation of choroidal vascular resistance to hemodynamic stress may lead to an increased choroidal blood flow and stress to the RPE cells in patients with hyperactivity of the sympathetic system. The patient's elevated blood pressure may, therefore, contribute to the development of PNV in this case [10]. Thus, the pathogenetic process leading to and maintaining the CNV is dependent on VEGF and might as well be affected by a dysregulation of choroidal vessels [11].

In the present case, we report on the first successful combination therapy of intravitreal anti-VEGF and oral off-label spironolactone for pachychoroid-associated CNV mimicking neovascular AMD. In spite of the marked disease chronicity and a long previous therapy of 29 intravitreal anti-VEGF injections, the addition of spironolactone for the first time allowed for a complete resolution of the previously recalcitrant subretinal fluid.

Spironolactone and eplerenone, both glucocorticoid-mineralocorticoid inhibitors, have been recently established as efficient off-label therapies for CSC [12]. Both facilitate subretinal fluid resolution and induce choroidal thinning by competitive inhibition of glucocorticoid signaling which is pathologically increased in CSC due to high endogenous ("stress-related") or exogenous glucocorticoid levels, which, due to the biochemical similarity of glucocorticoid and mineralocorticoid receptors, can activate mineralocorticoid signaling [12]. In turn, an overactivation of mineralocorticoid signaling has been shown to induce angiogenesis and choroidal thickening, while the mineralocorticoid aldosterone has been shown to induce choroidal thickening secondary to an upregulation of a vasodilatory potassium channel only present in the choroid [4].

Both in pachychoroid disorders and AMD, the role of subretinal fluid, especially when subfoveal, is complex and not fully elucidated. Commonly, disease activity of uncomplicated CSC is defined by subretinal fluid presence, and patients with nonresolving fluid are at high risk of vision-threatening retinal atrophy in the long-term [5]. In neovascular AMD, however, eyes with subretinal fluid have been shown to present better visual acuity than eyes with completely dry maculae, and subretinal fluid is being discussed as a biomarker protective of geographic atrophy [13]. In the case of CNV complicating pachychoroid disorders, for example, CSC or PNV, the case is even more complex, as subretinal fluid can both derive from CNV leakage (due to less aggressive CNV activity often faint to hardly discernible) or pachychoroid activity. For these reasons, at the moment no clear recommendations as to whether or not to completely dry maculae affected by pachychoroid maculopathies or neovascular AMD can be given from a long-term perspective.

Interestingly, the commencement of spironolactone and the withdrawal of anti-VEGF treatment showed a marked change in CNV morphology. CNV in pachychoroid disorders, namely CSC and PNV, is almost exclusively type 1 lesions [3]. In our case, the appearance of subretinal hyper-reflective material above the RPE was noted 6 weeks after the therapeutic intervention, not proving, but indicative of a type $2 \mathrm{CNV}$ conversion. It is unclear whether this is due to the withdrawal of anti-VEGF, leading to CNV regrowth, or the influence of spironolactone on the vasculature. Theoretically, thinning of the Haller layer induced by mineralocorticoid inhibition should improve choriocapillaris perfusion and thus reduce choroidal ischemia which is hypothesized to be a driving factor in pachychoroid disease. As recently reported by Jung et al. [14], long anti-VEGF treatment, however, causes a cumulative reduction of subfoveal choroidal thickness that is greater in Aflibercept than in ranibizumab treated eyes. For this reason, the influence of spironolactone on choroidal thickness in our pre-treated

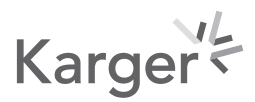


case should be expected to be lower than in a treatment-naïve eye. Mechanistically, the vascular stimulus of the mineralocorticoid antagonist might have caused an inhibition of the type $1 \mathrm{CNV}$ component below the RPE, while residual VEGF-levels in the vitreous body might have led to supra-RPE growth. A limitation regarding multimodal imaging was the fact, that at the time of CNV conversion into a type II CNV, only OCT-B scans were performed. An angiography to fully confirm type II CNV will be performed during the follow-up.

\section{Conclusion}

Pachychoroid disease should be considered in eyes with presumed AMD not adequately responding to anti-VEGF therapy. On OCT, measurements of subfoveal choroidal thickness and the absence of Drusen (especially on the often less affected partner eye) are easily recognizable guiding features. Spironolactone might represent a valuable additional therapy targeting choroidal malfunction in these cases and should be further examined as an adjunct therapy in randomized, controlled trials.

\section{Statement of Ethics}

This case report was approved by the IRB and the patient provided written consent for these data to be published.

\section{Conflict of Interest Statement}

Leonie Keidel received previous travel expenses from Roche Pharma. Jakob Siedlecki received income from honoraria as a lecturer from Novartis Pharma GmbH (Nürnberg, Germany), Pharm-Allergan GmbH (Frankfurt am Main, Germany), Carl Zeiss Meditec AG (Jena, Germany), and Oculentis OSD Medical GmbH (Berlin, Germany). Jakob Siedlecki received personal consultation fees from Bayer Pharma AG (Berlin, Germany). Jakob Siedlecki received travel reimbursement from Roche AG (Grenzach-Wyhlen, Germany). Benedikt Schworm received previous speaker fees and travel expenses from Novartis Pharma GmbH and Topcon Corporation. Siegfried Priglinger received previous speaker fees and/or travel expenses from Novartis Pharma GmbH, Oertli AG, Bayer AG, Alcon Pharma. GmbH and PharmAllergan $\mathrm{GmbH}$.

\section{Funding Sources}

No funding or grant support.

\section{Author Contributions}

Leonie Keidel authored the article, created the figures, and examined the patient. Jakob Siedlecki authored the article, created the figures, and examined the patient. Benedikt Schworm examined the patient and proofread. Siegfried Priglinger proofread.

\section{Karger's}


Keidel et al.: Combination Therapy for Pachychoroid Neovasculopathy Disguising as AMD

\section{Patient Consent}

Written consent to publish personal information and case details has been obtained from the patient. All research and measurements followed the tenets of the Declaration of Helsinki.

\section{Authorship}

All authors attest that they meet the current ICMJE criteria for authorship.

\section{References}

1 Yang S, Zhao J, Sun X. Resistance to anti-VEGF therapy in neovascular age-related macular degeneration: a comprehensive review. Drug Des Devel Ther. 2016;10:1857-67.

2 Fung AT, Yannuzzi LA, Freund KB. Type 1 (sub-retinal pigment epithelial) neovascularization in central serous chorioretinopathy masquerading as neovascular age-related macular degeneration. Retina. 2012;32(9): 1829-37.

3 Cheung CMG, Lee WK, Koizumi H, Dansingani K, Lai TYY, Freund KB. Pachychoroid disease. Eye. 2019;33(1): 14-33.

4 Padron-Perez N, Arias L, Rubio M, Lorenzo D, Garcia-Bru P, Catala-Mora J, et al. Changes in choroidal thickness after intravitreal injection of anti-vascular endothelial growth factor in pachychoroid neovasculopathy. Invest Ophthalmol Vis Sci. 2018;59(2):1119-24.

5 Mrejen S, Balaratnasingam C, Kaden TR, Bottini A, Dansingani K, Bhavsar KV, et al. Long-term visual outcomes and causes of vision loss in chronic central serous chorioretinopathy. Ophthalmology. 2019;126(4):576-88.

6 Dansingani KK, Gal-Or O, Sadda SR, Yannuzzi LA, Freund KB. Understanding aneurysmal type 1 neovascularization (polypoidal choroidal vasculopathy): a lesson in the taxonomy of "expanded spectra": a review. Clin Exp Ophthalmol. 2018;46(2):189-200.

7 Cheung CMG, Yanagi Y, Akiba M, Tan A, Mathur R, Chan CM, et al. Improved detection and diagnosis of polypoidal choroidal vasculopathy using a combination of optical coherence tomography and optical coherence tomography angiography. Retina. 2019;39(9):1655-63.

8 Warrow DJ, Hoang QV, Freund KB. Pachychoroid pigment epitheliopathy. Retina. 2013;33(8):1659-72.

9 Querques G, Souied EH. Vascularized drusen: slowly progressive type 1 neovascularization mimicking drusenoid retinal pigment epithelium elevation. Retina. 2015;35(12):2433-9.

10 Lupidi M, Fruttini D, Eandi CM, Nicolò M, Cabral D, Tito S, et al. Chronic neovascular central serous chorioretinopathy: a Stress/Rest Optical Coherence Tomography Angiography Study. Am J Ophthalmol. 2020;211: 63-75.

11 Cheung CMG, Lee WK, Koizumi H, Dansingani K, Lai TYY, Freund KB. Pachychoroid disease. Eye. 2019;33(1): 14-33.

12 Bousquet E, Beydoun T, Rothschild PR, Bergin C, Zhao M, Batista R, et al. Spironolactone for nonresolving central serous chorioretinopathy: a Randomized Controlled Crossover Study. Retina. 2015;35(12):2505-15.

13 Jaffe GJ, Ying GS, Toth CA, Daniel E, Grunwald JE, Martin DF, et al. Macular morphology and visual acuity in year five of the comparison of age-related macular degeneration treatments trials. Ophthalmology. 2019;126(2): 252-60.

14 Jung BJ, Kim JY, Lee JH, Baek J, Lee K, Lee WK. Intravitreal aflibercept and ranibizumab for pachychoroid neovasculopathy. Sci Rep. 2019;9(1):2055. 